\title{
Social, health, education and economic effects of COVID-19 on adolescent girls in Kenya: Responses from the first round of adolescent data collection, August 2020-Kisumu
}

Population Council

Follow this and additional works at: https://knowledgecommons.popcouncil.org/departments_sbsr-pgy How does access to this work benefit you? Let us know!

\section{Recommended Citation}

"Social, health, education and economic effects of COVID -19 on adolescent girls in Kenya: Responses from the first round of adolescent data collection, August 2020-Kisumu," COVID-19 Research \& Evaluations brief. Nairobi: Population Council, 2020. 


\title{
Social, health, education and economic effects of COVID -19 on adolescent GIRLS in Kenya.
}

\author{
Responses from the first round of adolescent data collection on \\ August 2020. \\ Kisumu
}

\section{Highlights}

Adolescent knowledge of COVID-19 risk factors, symptoms and preventive behaviors in Kisumu was high. Over half (59\%) knew asymptomatic transmission was possible. Perceived risk is mixed; those who perceive their risk as high mainly attribute this to interacting with a lot of people each day, higher for boys (25\%) than girls (16\%).

Almost all $(91 \%)$ adolescents said they had been doing some learning or school work from home, mainly reading other books $(53 \%)$, reading materials from school $(28 \%)$, or television/radio lessons (19\%). The main reason for not doing any was that the school hasn't provided any lessons (38\%) or trouble accessing the lessons provided (23\%).

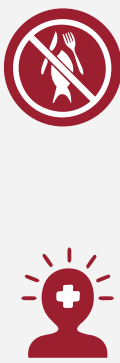

Food insecurity is a major threat: Many participants have been skipping meals or eating less since the COVID-19 pandemic began with $47 \%$ reporting they are skipping meals a couple times per week, and $79 \%$ say this is more so than before COVID-19. Before schools closed, $68 \%$ said they were receiving at least one meal at school. This was higher for older adolescents, and highest for older adolescent girls (72\%).

Mental health is a concern, as school closures become prolonged. Many respondents (47\%) have felt down or depressed in the last week, Over half very much agreed COVID-19 made them feel threatened or concerned (64\%) and that they were worried they or someone they love would get COVID-19 (62\%).

\section{Knowledge, Attitudes, and Perceived Risk of Infection}

Between August 15th-18th, 2020 a COVID-19 phone survey was completed with 603 10-19 year old adolescents in Kisumu, Kenya. The average age was 17 years, $71 \%$ were female and $39 \%$ have their own cell phone. Households were part of the DREAMS program and evaluation cohort.. Kisumu county is a port city on Lake Victoria and as of August 2020, there were 66 confirmed cases of COVID-19 in Kisumu.

- Knowledge: Overall, respondents in Kisumu correctly identified the elderly as at high risk if infected (63\%) and those with weakened immune systems $(27 \%)$, although half incorrectly stated children are at highest risk (53\%). Knowledge of fever (78\%), cough (84\%) and difficulty breathing (57\%) as symptoms was high. Over half (59\%) knew asymptomatic transmission was possible. Almost all (86\%) knew that people of all ages can become infected. A small proportion believed COVID-19 cannot spread in hot places (12\%) and that it is a punishment from god (17\%).

- Prevention: Most adolescents (95\%) correctly identified handwashing as a method to prevent the spread of COVID-19 and over three-quarters identified wearing masks (83\%) and standing 1 to 2 meters apart $(80 \%)$ as prevention methods.

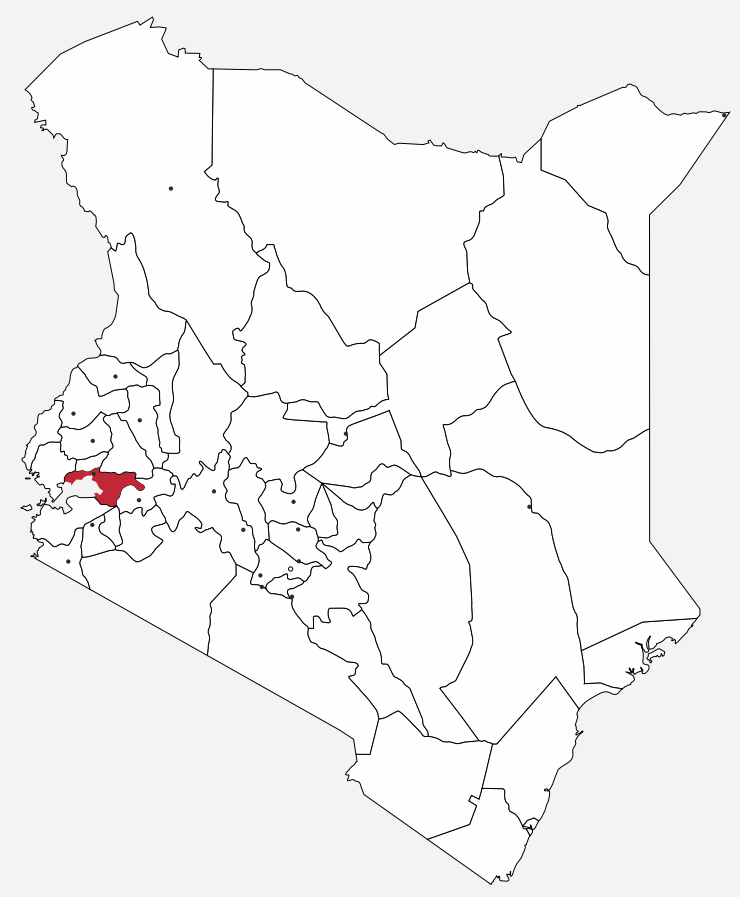


- Information source: Over $80 \%$ of adolescents reported receiving information on COVID-19 from TV or radio (Government or regular programming), only $47 \%$ said from government SMS, and $68 \%$ said from friends. Over half $(63 \%)$ said from their parents.

- Perceived risk: Almost half of adolescents (45\%) believed their risk of contracting COVID-19 was low. Those with low perceived risk say it is because they have been washing their hands or using sanitizer (43\%), wearing a face mask (40\%), and staying at home 37\%). Only $2 \%$ knew someone who had tested positive for COVID-19.

\section{COVID-19 Prevention Education and Behavior}

- Most adolescents left home at least once in the past week (81\%), of those that went out, $97 \%$ wore a mask, $95 \%$ washed their hands upon returning home, and $85 \%$ kept a distance of 1-2 meters. However, of those that went out, half said they interacted with five or more people - this was higher for males than females.

- About a third (32\%) said they rode public transportation when they went out; this was higher for older adolescents, and highest for females ages $15-19$ years old ( $41 \%$, vs $24 \%$ of males the same age).

\section{Impact of the COVID-19 Mitigation Response}

The COVID-19 pandemic and associated physical distancing and lockdown measures have had a large economic, health, educational, and social impact on respondents. Although schools have been closed since March 2020, many adolescents were able to access school-work. Girls in particular faced an increase in domestic chores, and some older adolescents were working, threatening returns to school when they reopen.

\section{Education and Income Generating Activities.}

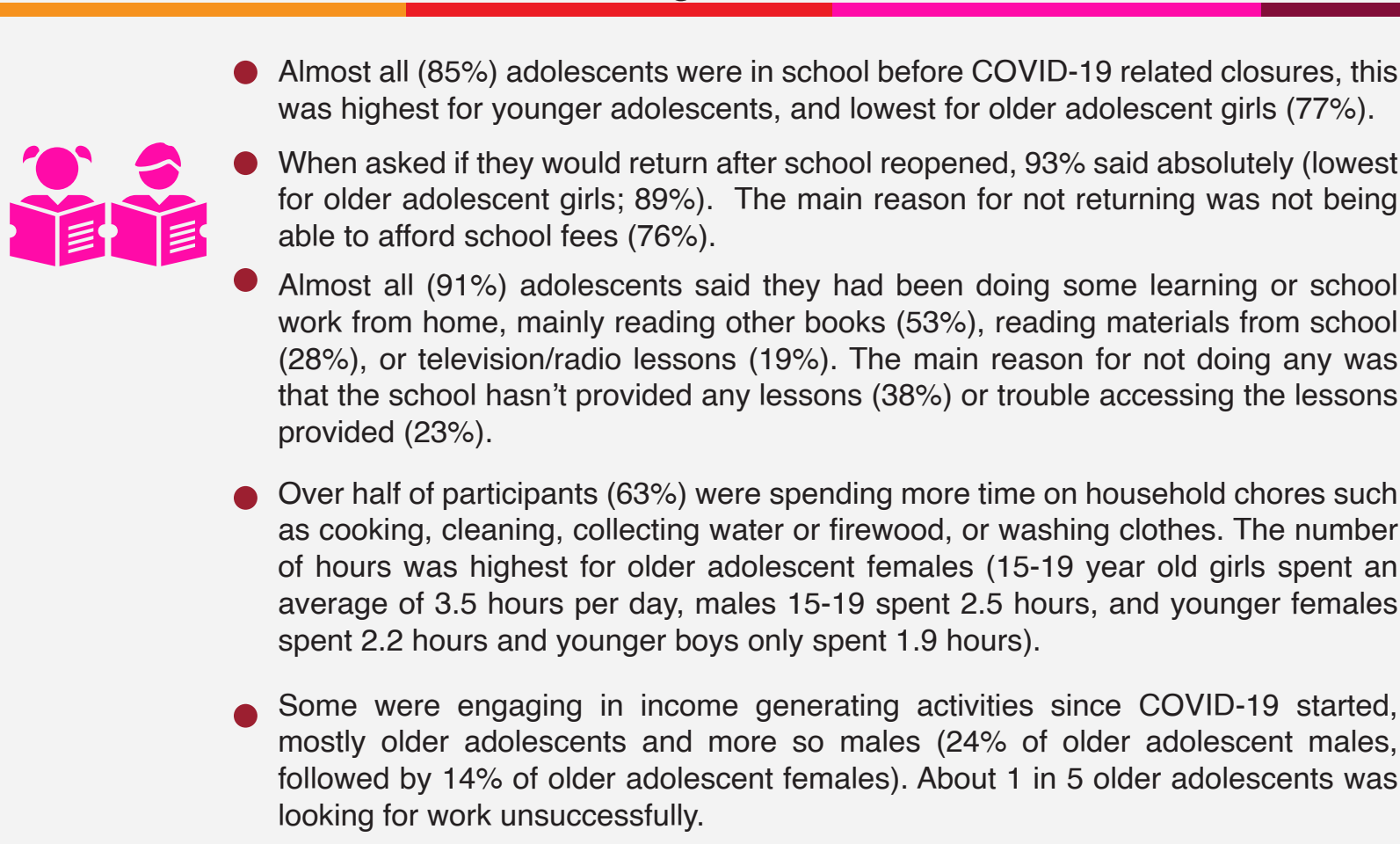




\section{Decision Making}

- Compared to boys, girls have been spending more time on household chores since COVID-19 began ( $63 \%$ vs $41 \%)$. This links to girls reporting spending more time at home than boys ( $86 \%$ v. $55 \%)$.

- When asked whether they have more, less, or the same amount of control over when to leave the house compared to before COVID-19, most boys reported having less control $(54 \%)$ while girls reported having more control $(50 \%)$.

\section{Food Security}

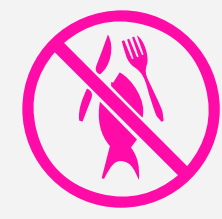

- Many participants have been skipping meals or eating less since the COVID-19 pandemic began. In the past 7 days, $47 \%$ said they are skipping meals a couple times per week, and $79 \%$ say this is more often than before COVID- 19 .

- Before schools closed, $68 \%$ said they were receiving at least one meal at school. This was higher for older adolescents, and highest for older adolescent girls (72\%).

\section{Health and Safety}

- Almost half of participants reported feeling down, depressed or hopeless, or feeling little interest or pleasure in things they normally do (47\%). This was higher for older adolescents, highest for adolescent males.

- Of girls who have started menstruating, disposable sanitary pads are the most commonly used product, and over half $(54 \%)$ say they are having challenges getting their preferred product, mainly due to them or their parents not being able to afford it.

- About $12 \%$ of participants reported having been humiliated, insulted or threatened in the past one month, highest for males than females, and highest for youngest males (19\% of males $10-14$ years of age). About $8 \%$ overall reported having been hit, slapped, kicked or beaten in the last one month, highest for younger adolescents (16\% of younger adolescent boys, $13 \%$ of younger adolescent girls).

- $94 \%$ of girls are not currently pregnant and do not wish to get pregnant, $2 \%$ were pregnant or probably pregnant. Male condoms were the most commonly reported contraceptive method used. Over a third (39\%) were currently doing something to avoid or delay pregnant. The main reason for not using a method was that the participant is not currently sexually active. 


\section{Conclusions and Recommendations}

COVID-19 public education campaigns in Kisumu should continue with a focus on:

- As knowledge of symptoms and transmission was relatively high, most adolescents still say there interacting with a lot of people when they go out. Ensuring mask wearing, handwashing, and staying home unless urgent is critical to reducing transmission.

Address the health, economic, and social impacts of lockdowns:

- Ensuring access to educational materials at home while schools are closed is critical, with attention to older adolescent girls who may be more likely to fall behind.

- Adolescents are missing meals, especially since they are also not receiving meals from school during closures. Food distributions are necessary, targeting older adolescents who were receiving more meals at school prior to closures.

- Address mental health, as many adolescents say they feel depressed or down due to the pandemic, and many report anxiety about themselves or someone they love getting COVID-19. 